Research Paper

\title{
Circular RNA CCDC66 promotes gastric cancer progression by regulating c-Myc and TGF- $\beta$ signaling pathways
}

Guifang $\mathrm{Xu}^{1,2 \#,}$, Yanke Chen#, Min Fu' ${ }^{1,2}$, Xueyan Zang2, Mingming Cang ${ }^{1,2}$, Yanlong Niu1,2, Weiya Zhang ${ }^{2}$, Yu Zhang2, Zheying $\mathrm{Mao}^{2}$, Meng Shao ${ }^{2}$, Hui Qian², Wenrong $\mathrm{Xu}^{2}$, Hui Cai ${ }^{\circledR 凶}$, Pengcheng Jiang ${ }^{\bowtie}, \mathrm{Xu}$ Zhang $2,3{ }^{\boxplus}$

1. Institute of Digestive Diseases, The Affiliated People's Hospital of Jiangsu University, Zhenjiang, Jiangsu 212002, China

2. Jiangsu Key Laboratory of Medical Science and Laboratory Medicine, School of Medicine, Jiangsu University, Zhenjiang, Jiangsu 212013, China

3. Key Laboratory of Molecular Diagnostics and Precision Medicine for Surgical Oncology in Gansu Province, Gansu Provincial Hospital, Gansu 730000, China

\# These authors contributed equally to this work.

$\triangle$ Corresponding authors: Xu Zhang, Pengcheng Jiang, or Hui Cai, Jiangsu Key Laboratory of Medical Science and Laboratory Medicine, Jiangsu University, 301 Xuefu Road, Zhenjiang, Jiangsu 212013, China. Tel: +86 511 85038215; Fax: +86 511 85038449; E-mail: xuzhang@ujs.edu.cn (X. Zhang), jpc18906106623@126.com (P. Jiang) or caialon@163.com (H. Cai).

(C) The author(s). This is an open access article distributed under the terms of the Creative Commons Attribution License (https://creativecommons.org/licenses/by/4.0/). See http://ivyspring.com/terms for full terms and conditions.

Received: 2019.06.19; Accepted: 2020.02.06; Published: 2020.02.20

\begin{abstract}
Background: CircRNAs play important roles in cancer development and progression and have the potential to serve as cancer biomarkers. The aim of this study was to investigate the role of circular RNA CCDC66 (circCCDC66) in gastric cancer and to reveal the underlying mechanisms.

Methods: The expression of circCCDC66 in GC tissues and cell lines was examined by qRT-PCR. The correlation between circCCDC66 expression level and clinicopathological characteristics was analyzed. The biological roles of circCCDC66 in GC cell apoptosis, proliferation, migration and invasion were determined by flow cytometry, cell counting, cell colony formation, wound healing, transwell migration and matrigel invasion assays. The role of circCCDC66 in GC growth was further confirmed by mouse xenograft tumor model. Western blot and qRT-PCR were used to explore the effects of circCCDC66 on epithelial-mesenchymal transition (EMT)-related gene and protein expression.
\end{abstract}

Results: CircCCDC66 expression was elevated in both GC tissues and cell lines compared to adjacent normal tissues and normal gastric epithelial cell line. The upregulation of circCCDC66 in GC tissues was related to tumor stage and lymphatic metastasis. CircCCDC66 knockdown significantly inhibited GC cell proliferation, migration and invasion and induced cell apoptosis in GC cells. On the contrary, circCCDC66 overexpression had the opposite effects. In addition, circCCDC66 knockdown suppressed the tumorigenesis of GC cells in nude mice. Furthermore, circCCDC66 knockdown inhibited the activation of c-Myc and TGF- $\beta$ signaling pathways and reversed EMT in GC cells. c-Myc and TGF- $\beta$ interference blocked circCCDC66-mediated promotion of gastric cancer cell proliferation, migration and invasion.

Conclusion: CircCCDC66 promotes GC growth and metastasis by activating c-Myc and TGF- $\beta$ signaling pathways, suggesting that it may serve as a potential biomarker for GC.

Key words: circular RNA; gastric cancer; circCCDC66; EMT; progression

\section{Introduction}

Gastric cancer (GC) is one of the most common cancers worldwide with high morbidity and mortality $[1,2]$. Although many advances have been made in the diagnosis and treatment of this disease, the prognosis of GC patients remains poor with a 5-year overall survival less than $30 \%$ in most countries [3]. 
Therefore, there is an urgent need to further understand the molecular mechanism for GC development and progression and to seek for biomarkers for GC early diagnosis and prognosis prediction.

Circular RNAs (circRNAs) are noncoding RNAs that are covalently linked to form a closed circular structure without $5^{\prime}$ caps and $3^{\prime}$ tails [4,5]. CircRNAs display cell or tissue specific expression and are conserved across species due to their resistance to RNase R. Compared to their linear counterparts, circRNAs are highly stable and accumulate in both nucleus and cytoplasm, indicating important roles in human health. CircRNAs participate in a wide range of biological processes, including transcription [6], mRNA splicing [7], RNA decay and translation [8], and their dysregulation leads to abnormal cellular functions and human diseases, such as cardiovascular diseases [9], temporal lobe epilepsy [10], Alzheimer's disease [11], metabolic diseases [12], and cancer [13]. Emerging evidence suggest that some circRNAs act as miRNA sponges [14,15] and interact with RNA binding proteins (RBPs) [16,17] to modulate gene expression. For instance, ciRS-7 serves as miR-7 sponge to regulate the expression of several oncogenes [18] and circHIPK3 as miR-124 sponge to suppress cell proliferation in multiple cancers [19].

Recent studies suggest that circRNAs are deregulated in various cancers, including hepatocellular cancer (HCC) [20], colorectal cancer (CRC) [21], esophageal squamous cancer [22], and breast cancer [23]. CircRNAs are involved in cancer growth, metastasis, and therapy resistance [24,25]. CircRNAs have also been shown to play important roles in gastric cancer proliferation, migration and metastasis and can serve as diagnostic biomarkers [26]. Zhang et al. demonstrate that the expression of circLARP4 is downregulated in GC tissues and circLARP4 represents an independent prognostic factor for overall survival of GC patients [27]. Liu et al. demonstrate that circYAP1 functions as a tumor suppressor in GC cells by targeting the miR-367-5p/p27 axis and it provides a prognostic indicator of survival in GC patients [28]. Yang et al. demonstrate that circ-CTNNB1 activates $\beta$-catenin signaling to promote gastric cancer progression through DDX3-mediated transactivation of YY1 [29]. These findings suggest that circRNAs are crucial for gastric cancer development and progression and have great promise to be used as biomarkers and targets for gastric cancer diagnosis and therapy.

Circular RNA CCDC66 (circCCDC66) was previously found to be highly expressed in human colorectal cancer and could promote CRC growth and metastasis [21]. However, its role in gastric cancer has not been reported yet. In this study, we found that the expression of circCCDC66 was increased in gastric cancer tissues and cell lines, and its expression level was associated with tumor stage and lymphatic metastasis. CircCCDC66 knockdown inhibited while circCCDC66 overexpression promoted GC growth and metastasis by inducing epithelial to mesenchymal transition (EMT) through the activation of c-Myc and TGF- $\beta$ signaling pathways.

\section{Materials and Methods}

\section{Patients and samples}

A total of 70 patients with gastric cancer who underwent gastrectomy at the First People's Hospital of Zhenjiang between November 2015 and October 2017 were enrolled in this study. The adjacent non-cancerous tissues were collected at $5 \mathrm{~cm}$ away from the tumor site. Tumor stages were classified following the $8^{\text {th }}$ edition tumor-node-metastasis (TNM) classification system of the American Joint Committee on Cancer. Histological grade was assessed by two experienced pathologists. All the patients enrolled in this study had not received any radiation or chemotherapy and had no other cancers before. This study was approved by Institutional Reviewing Board of First People's Hospital of Zhenjiang and informed consent was obtained from each patient.

\section{Primer design and synthesis}

The primer for circCCDC66 and small molecular RNA U6 was synthesized by Hanbio Biotechnology (Shanghai, China). The sequences of circCCDC66 and U6 were as follows: circCCDC66: F: ACCTACAAC CGGAAGCCAG, R: AGCAGTACTGTTTCCTGATG C; U6: F: CTCGCTTCGGCAGCACA, R: AACGCTTC ACGAATTTGCGT.

\section{Quantitative real-time polymerase chain reaction (qRT-PCR)}

Total RNA was extracted from tissues and cells by Trizol reagent (Invitrogen) and quantified by NanoDrop Spectrophotometer. Total RNA was reverse transcribed to cDNA by using HiScript 1st Strand cDNA Synthesis Kit (Vazyme, Nanjing, China). The cDNA samples were quantified on CFX96 Real-time PCR Detection System (Bio-Rad, Hercules, CA, USA) by using UltraSYBR Mixture (CWBIO, Beijing, China) according to the manufacturer's instructions. U6 was used as internal control.

\section{Cell culture}

Human gastric cancer cell lines BGC-823, MGC-803, SGC-7901, and HGC-27 were purchased from the Shanghai Institute of Biochemistry and Cell 
Biology, Chinese Academy of Sciences (Shanghai, China). Human normal gastric epithelial cell line GES-1 was obtained from Gefan Biological Technology (Shanghai, China). BGC-823, SGC-7901, HGC-27, and GES-1 cells were routinely cultured in RPMI 1640 medium (Invitrogen, Carlsbad, CA, USA) supplemented with $10 \%$ fetal bovine serum (FBS) (Gibco). MGC-803 cells were cultured with high glucose DMEM (Invitrogen). All the cell lines were cultured at $37^{\circ} \mathrm{C}$ humidified atmosphere containing $5 \% \mathrm{CO}_{2}$.

\section{Cell transfection}

Control and circCCDC66 siRNAs were synthesized by Hanbio Biotechnology (Shanghai, China). The sequences of siRNAs were as follows: si-circCCDC66: 5'-CAAUUAGAGCAUCAGGAAAT T-3'; si-control: 5'-UUCUCCGAACGUGUCACGUTT3'; si-TGF- $\beta 1$ : 5'-GCAGAGUACACACAGCAUATT3'; si-c-Myc: GUGCAGCCGUAUUUCUACUTT. GC cells $\left(2 \times 10^{5} /\right.$ well $)$ in logarithmic growth period were transfected with overexpressing plasmid and silencing siRNAs by using LipoFiter (Hanbio, Shanghai, China) according to the manufacturer's instructions. Cells were collected at 36 hours after transfection. The transfection efficiency was assessed by qRT- PCR.

\section{Cell counting assay and colony formation assay}

At 24 hours after transfection, the cells were collected and plated into 24-well plates at $10^{4}$ cells/well. The number of cells were counted every day for consecutive 6 days. For colony formation assay, the transfected cells $\left(10^{3}\right.$ cells/well) were plated into the 6-well plates and cultured for consecutive 10 days. The colonies were fixed with polyformaldehyde and stained with $0.1 \%$ crystal violet.

\section{Cell migration and invasion assays}

For cell migration assay, the transfected cells $\left(2 \times 10^{4}\right.$ cells/well $)$ were suspended in serum-free medium and plated on the upper chamber of transwell with $8 \mu \mathrm{m}$ size pore (Corning, NY, USA). For cell invasion assay, the transfected cells $\left(1 \times 10^{5}\right.$ cells/well $)$ were placed in the upper chamber of transwell with $50 \mu \mathrm{l}$ matrigel coated. The lower chamber of transwell was filled with $600 \mu 1$ RPMI 1640 medium containing 10\% FBS. After incubation for 36 $h$, the cells on the surface of upper chamber were removed by scraping with a cotton swab, while the cells on the lower surface of the inserts were fixed with polyformaldehyde for $30 \mathrm{~min}$ and stained with $0.1 \%$ crystal violet for $15 \mathrm{~min}$. After that, each insert was counted under an inverted microscope (Olympus, Tokyo, Japan).

\section{Wound healing assay}

The transfected cells were plated in 6-well plates $\left(4 \times 10^{5}\right.$ cells / well). Wounds were created by using a $100 \mu 1$ pipette tip at 24 hours after inoculation. Then, the floating cells and debris were washed out by PBS. Wound healing was observed at different time points and the control group and experimental group were photographed at the same time.

\section{Cell apoptosis assay}

Cells were harvested at 36 hours after transfection and then stained with Annexin Alexa Fluor 647 and PI at room temperature in dark for 15 min. FACSCalibur flow cytometry (BD Biosciences, San Jose, CA, USA) was used to evaluate the percentage of apoptotic cells. Finally, Cell Quest Software was used to analyze the data.

\section{Western Blot}

Proteins were extracted with Radio-Immunoprecipitation Assay (RIPA) extraction reagent (Beyotime, Beijing, China). Equal amounts of protein were separated via $12 \%$ polyacrylamide gel, then switched to PVDF membrane (Millipore, Billerica, MA, USA). After being blocked with 5\% non-fat milk for $1 \mathrm{~h}$, the PVDF membranes were incubated with specific primary antibodies (Cell Signaling Technology, Shanghai, China) including p21, poly(ADP-ribose) polymerase (PARP), caspase-3, Bcl-2, E-cadherin, N-cadherin, Slug, Vimentin, c-Myc, TGF- $\beta$, p-SMAD2, SMAD2 and MMP9 antibodies. GAPDH (Sigma-Aldrich, St. Louis, MO, USA) was used as the internal control. Afterwards, the membrane was incubated with secondary antibodies (CST) for $2 \mathrm{~h}$. Finally, protein bands were visualized by using chemiluminescence (Millipore).

\section{Mouse tumor model}

4-6 weeks old male athymic nude mouse were purchased from Nanjing Model Animal Center (Nanjing, China) and maintained in SPF condition with a standard 12-h light-dark cycle. SGC-7901 cells transfected with si-circCCDC66 and si-control were suspended in PBS $\left(6 \times 10^{6} / 200 \mu \mathrm{l}\right)$ and then subcutaneously injected into the mice. Tumor growth was monitored twice per week. Tumor volume was evaluated using the formula $0.5 \times\left(\mathrm{a} \times \mathrm{b}^{2}\right)$, in which a means the long diameter, $b$ represents the short diameter. Four weeks later, the mice were sacrificed and the tumor tissues were harvested. The tumor tissues were weighed and fixed in $4 \%$ formalin for immunohistochemical analysis.

\section{Immunohistochemistry}

Tumor tissue sections were incubated with 
primary monoclonal antibody against Ki-67 followed by incubation with the secondary antibody for $30 \mathrm{~min}$ at room temperature. Afterwards, the sections were incubated with 3, 3'-Diaminobenzidine (3, 3'-DAB, Maxim, Fuzhou, China) for $5 \mathrm{~min}$, then counterstained with hematoxylin for $30 \mathrm{~s}$. Finally, the sections were photographed under a TE2000 microscope (Nikon, Tokyo, Japan).

\section{Statistical analysis}

All the data were expressed as mean \pm SD. Chi-square test or one-way analysis of variance (ANOVA) were conducted by using SPSS20.0 software (SPSS, Chicago, IL, USA) to compare the statistical differences. The results were considered statistically significant when $P$-value $<0.05$.

\section{Results}

\section{Circ CCDC66 is upregulated in gastric cancer tissues and cell lines}

We detected the expression of circCCDC66 in gastric cancer tissues and cell lines by using qRT-PCR. As shown in Figures $1 \mathrm{~A}$ and 1B, circCCDC66 was overexpressed in $68.5 \%(48 / 70)$ of $G C$ tissues compared to adjacent normal tissues $(P<0.001)$. We then analyzed the correlation between circCCDC66 expression level and clinicopathological parameters of GC patients. CircCCDC66 expression level was positively associated with tumor stage $(P=0.01)$ and lymphatic metastasis $(P=0.014)$ (Supplementary Table 1). Furthermore, circCCDC66 expression was also upregulated in GC cell lines compared to normal gastric epithelial cell line (Figure 1C). In summary, circCCDC66 was upregulated in GC tissues and cell lines and its expression level was associated with GC progression.

\section{CircCCDC66 knockdown inhibits and circCCDC66 overexpression promotes the proliferation of gastric cancer cells in vitro}

To determine the role of circCCDC66 in GC cell proliferation, we knocked down circCCDC66 in SGC-7901 and HGC-27 cells and overexpressed circCCDC66 in BGC-823 and HGC-27 cells by gene transfection. The efficiency of gene knockdown and gene overexpression was confirmed by qRT-PCR (Figure 2A). The results of cell counting assay showed that the number of cells in si-circCCDC66 group was less than that in si-control group at 48 hours after cell plating (Figure 2B). In addition, the number of cell colonies in si-circCCDC66 group was also significantly less than that in si-control group (Figure $2 \mathrm{C})$. On the contrary, the number of cells and cell colonies in circCCDC66 overexpressing group was significantly more than that in control group (Figures $2 B$ and $2 \mathrm{C}$ ).

\section{CircCCDC66 silencing inhibits gastric cancer growth in vivo}

We next wanted to know the role of circCCDC66 in GC growth in vivo. Athymic nude mouse were injected with si-control and si-circCCDC66 transfected SGC-7901 cells subcutaneously. As shown in Figures $3 \mathrm{~A}$ and $3 \mathrm{~B}$, the tumors in si-circCCDC66 group grew slowly compared to those in si-control group. At the end of experiment, the mean volume and weight of tumors in si-circCCDC66 group were smaller than that in si-control group (Figure $3 \mathrm{C}$ ). The results of immunohistochemical analysis showed that tumor tissues in si-circCCDC66 group had less Ki-67 positive cells than that in si-control group (Figure 3D). These data suggest that circCCDC66 could promote gastric cancer growth in vivo.
A

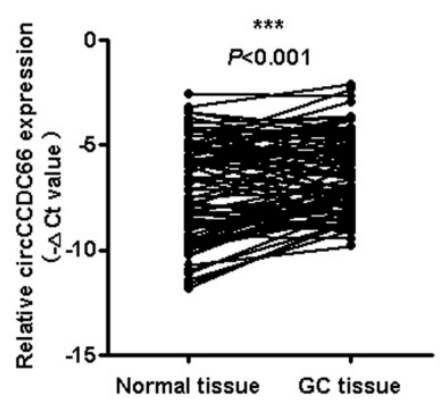

B

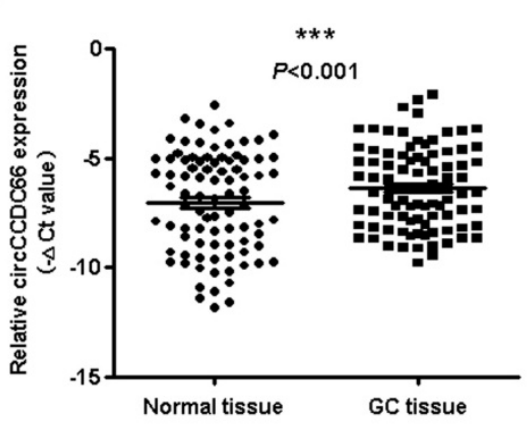

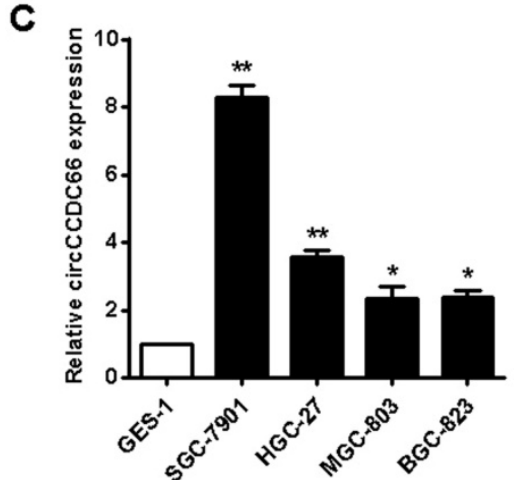

Figure 1 CircCCDC66 is upregulated in gastric cancer tissues and cell lines. (A and B) The expression levels of circCCDC66 in GC tissues and paired normal tissues $(n=70)$. (C) The expression profiles of circCCDC66 in SGC-7901, HGC-27, MGC-803, BGC-823 and GES-1 cell lines. The experiment was repeated for three times. $* P<0.05, * * p<0.01, * * * p<0.001$. 
A

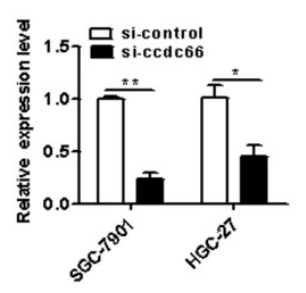

B
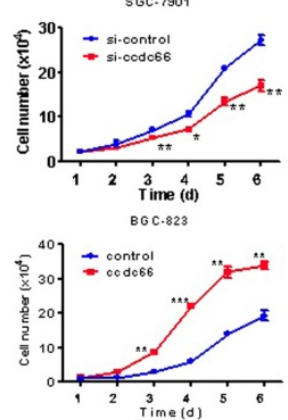
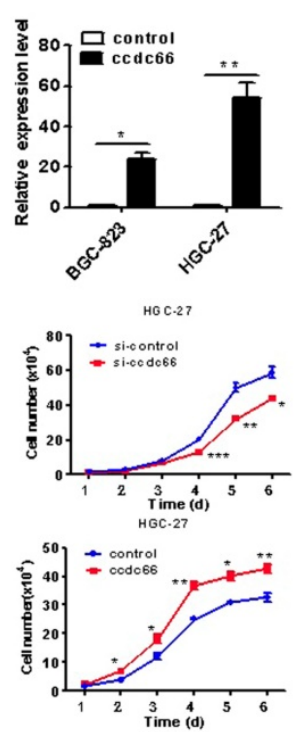

C
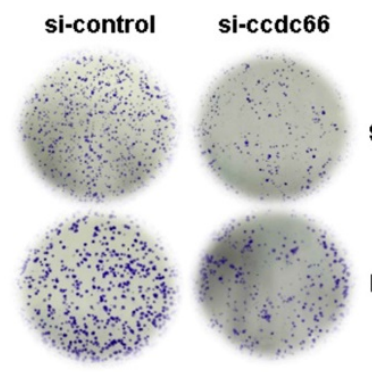

SGC-7901
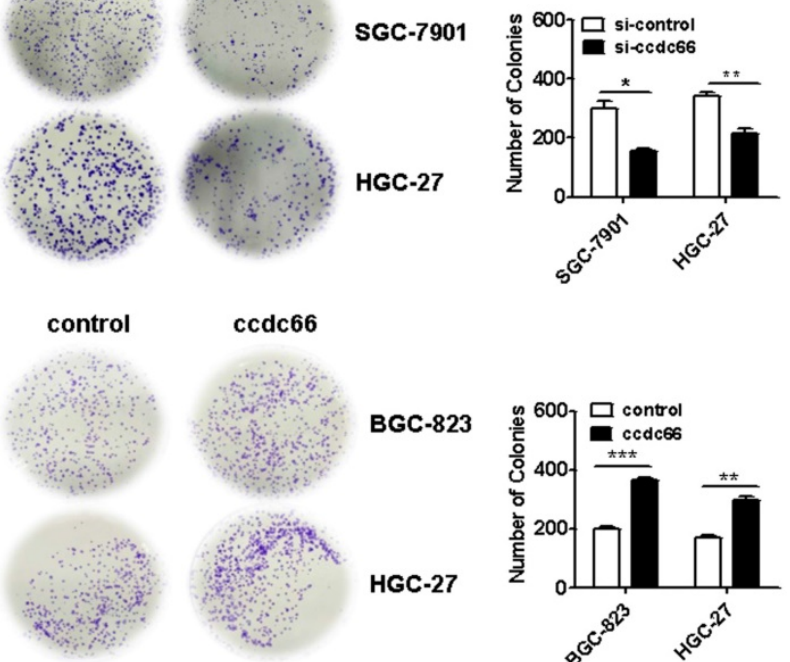

ccdc66

HGC-27

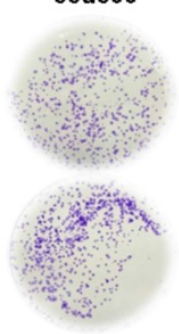

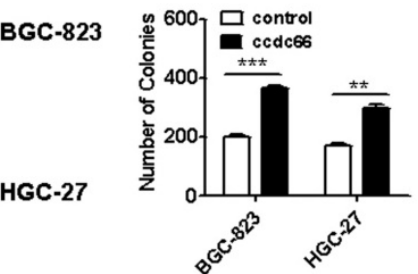

Figure 2 CircCCDC66 knockdown inhibits while overexpression promotes the proliferation of gastric cancer cells. (A) CircCCDC66 expression levels in siRNAs and overexpressing plasmid transfected GC cells. (B) Cell growth curves for circCCDC66 siRNAs and overexpressing plasmid transfected GC cells. (C) The colony formation ability of circCCDC66 siRNAs and overexpressing plasmid transfected GC cells. $* P<0.05, * * P<0.01, * * * P<0.001$.

A

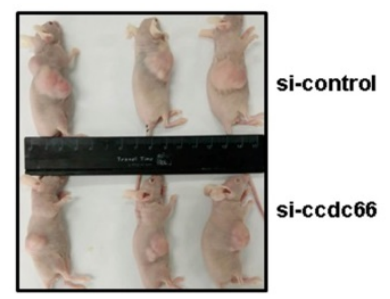

B

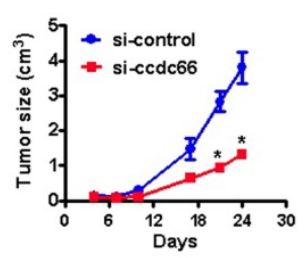

C
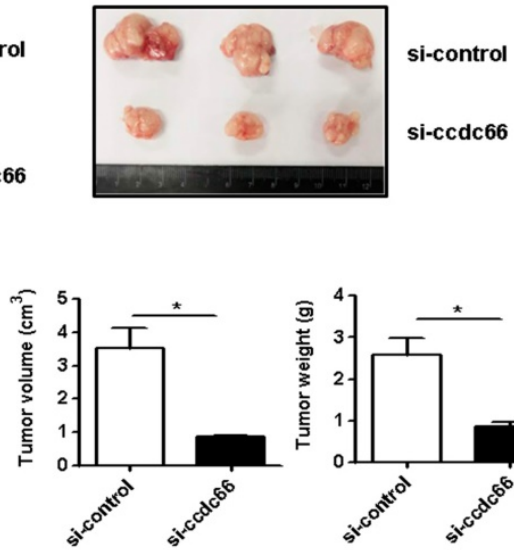

D

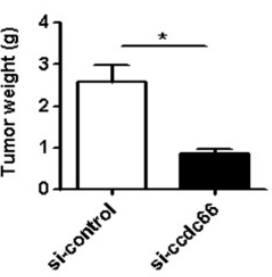

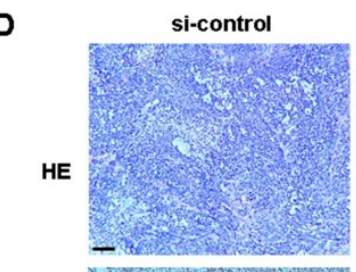

si-control

Ki-67

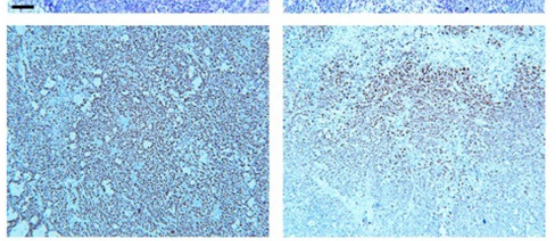

Figure 3 circCCDC66 knockdown inhibits gastric cancer growth in vivo. (A) Representative images of tumor-bearing mice and tumors in control and circCCDC66 knockdown groups. (B) Tumor growth curves of mice in control and circCCDC66 knockdown groups ( $n=3)$. (C) Tumor volumes and weights of mice in control and circCCDC66 knockdown groups $(n=3)$. (D) HE staining and immunohistochemical staining of Ki- 67 in mouse tumor tissues in control and circCCDC66 knockdown groups. Scale bar: $100 \mu \mathrm{m}$. $* p<0.05, * * p<0.01$, ***p $<0.001$.

\section{CircCCDC66 gene silencing induces cell apoptosis}

We further determined the effect of circCCDC66 on gastric cancer cell apoptosis. The results of flow cytometric analyses indicated that circCCDC66 knockdown promoted cell apoptosis in both SGC-7901 and HGC-27 cells (Figure 4A). Moreover, the results of qRT-PCR showed that circCCDC66 silencing inhibited the expression of $\mathrm{Bcl}-2$ but promoted the expression of Bax. The expression of Bcl-2 was upregulated and that of Bax downregulated in circCCDC66 overexpressing GC cells (Figure 4B). The results of western blot indicated that circCCDC66 knockdown promoted the expression of p21, cleaved
PARP and caspase-3 while inhibited that of Bcl-2. By contrast, circCCDC66 overexpression showed inverse changes in p21, cleaved PARP and Caspase-3, and Bcl-2 expression in GC cells (Figure 4C). The results suggest that circCCDC66 could inhibit GC cell apoptosis.

\section{CircCCDC66 knockdown inhibits while gene overexpression promotes GC cell migration and invasion}

The results of wound healing assay showed that circCCDC66 knockdown caused a notably lower scratch closure rate than that observed in control cells. CircCCDC66 overexpressing cells showed a higher 
scratch closure rate than control cells (Figure 5A). The results of transwell migration and matrigel invasion assays showed that circCCDC66 knockdown suppressed while circCCDC66 overexpression enhanced the migration and invasion abilities of GC cells (Figure 5B and 5C).

\section{CircCCDC66 regulates c-Myc and TGF- $\beta$ signaling pathways in GC cells}

Since circCCDC66 has been shown to affect cancer cell proliferation, migration and invasion and it has been reported to regulate several oncogenic genes including c-Myc, we performed qRT-PCR and western blot to explore the effect of circCCDC66 on the expression of c-Myc and TGF- $\beta$ signaling pathways in GC cells. As shown in Figure 6A,
circCCDC66 knockdown decreased the expression of $\mathrm{N}$-cadherin, Vimentin, Slug and c-Myc but increased the expression of E-cadherin in SGC-7901 and HGC-27 cells. CircCCDC66 overexpression enhanced the expression of $\mathrm{N}$-cadherin, Vimentin, Slug and c-Myc, while decreased that of E-cadherin in BGC-823 and HGC-27 cells. The results of western blot were consistent with that of qRT-PCR (Figure 6B). Moreover, we found that circCCDC66 knockdown inhibited the expression of TGF- $\beta$, p-SMAD2 and MMP9 while circCCDC66 overexpression had the opposite effects (Figure 6C). In summary, circCCDC66 could regulate GC progression by regulating c-Myc and TGF- $\beta$ signaling pathways.
A

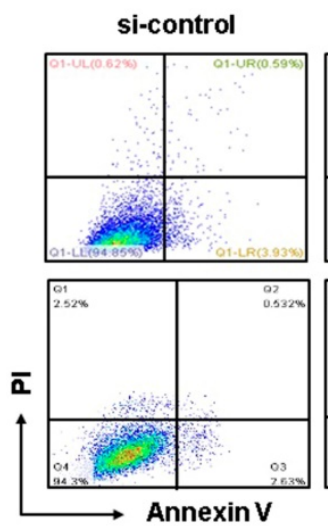

B

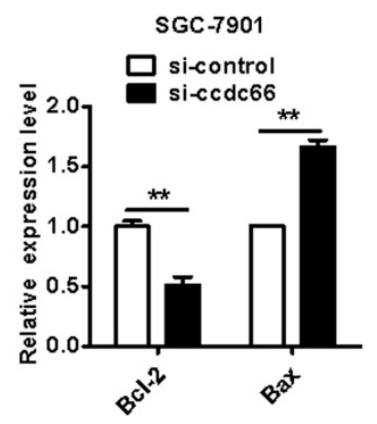

C

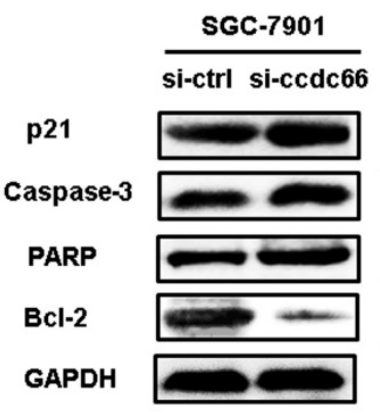

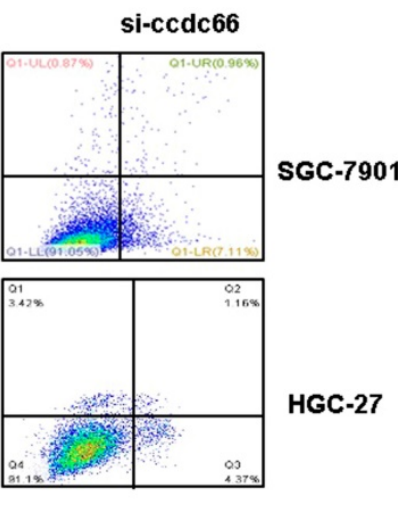

HGC-27

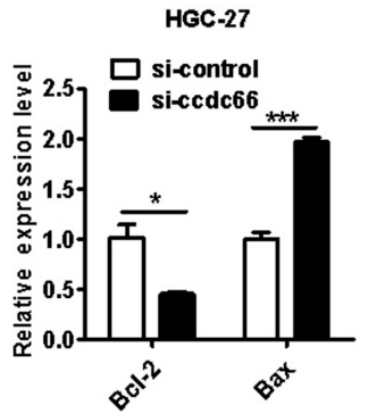

HGC-27
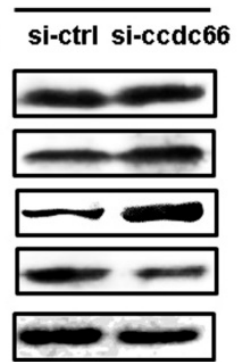

p21

Caspase-3

PARP

Bcl-2

GAPDH
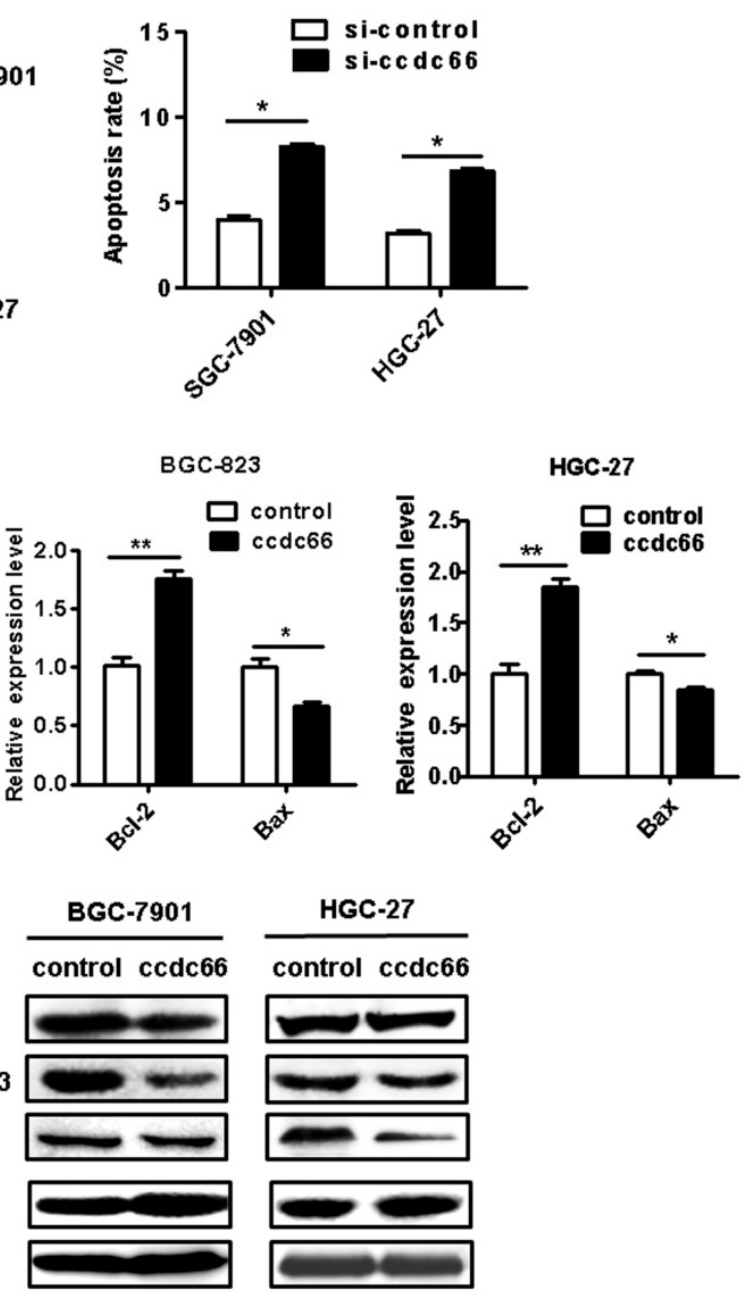

control ccdc66

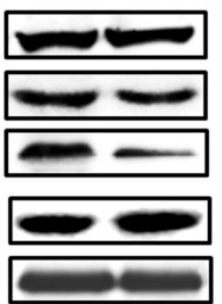

Figure 4 CircCCDC66 gene silencing suppresses while gene overexpression promotes the apoptosis of GC cells. (A) Flow cytometric analyses of cell apoptosis in control and circCCDC66 knockdown groups. (B) QRT-PCR analyses of Bcl-2 and Bax gene expression in circCCDC66 knockdown and overexpressing GC cells. (C) Western blot analyses of p21, poly (ADP- ribose) polymerase (PARP), Bcl-2 and Caspase-3 expression in circCCDC66 knockdown and overexpressing GC cells. All the experiments were repeated for three times. $* P<0.05$, $* * P<0.01$, $* * * P<0.001$. 
A
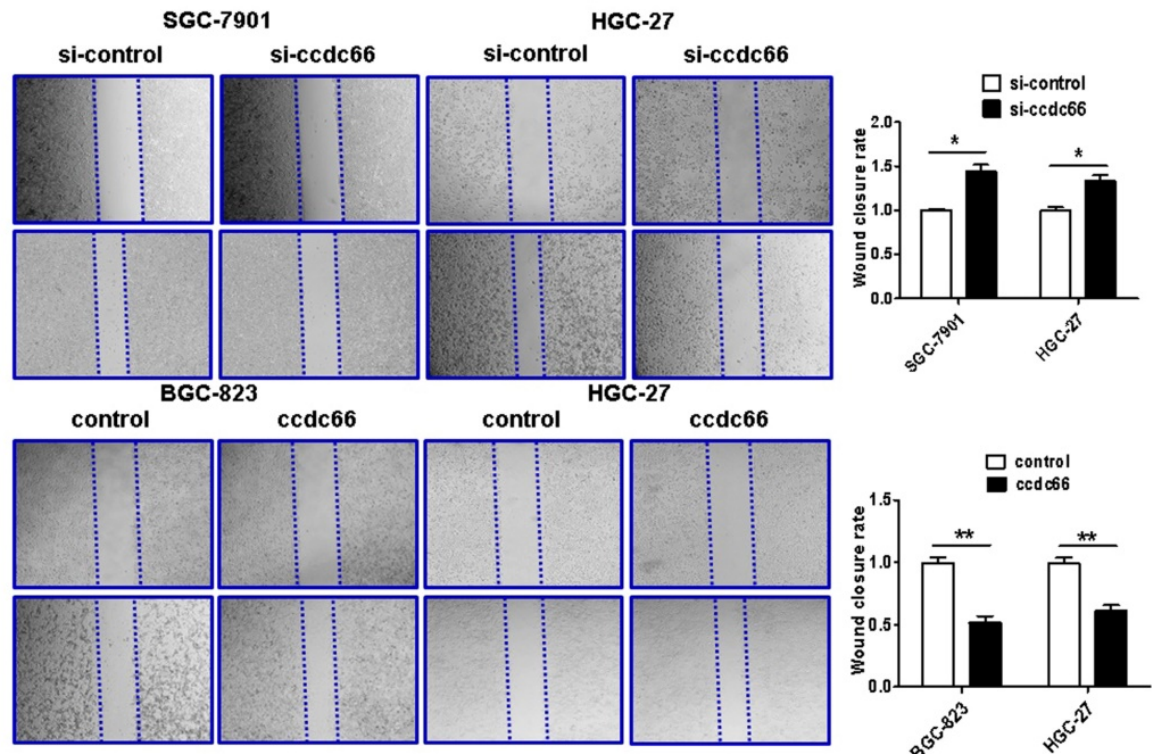

B
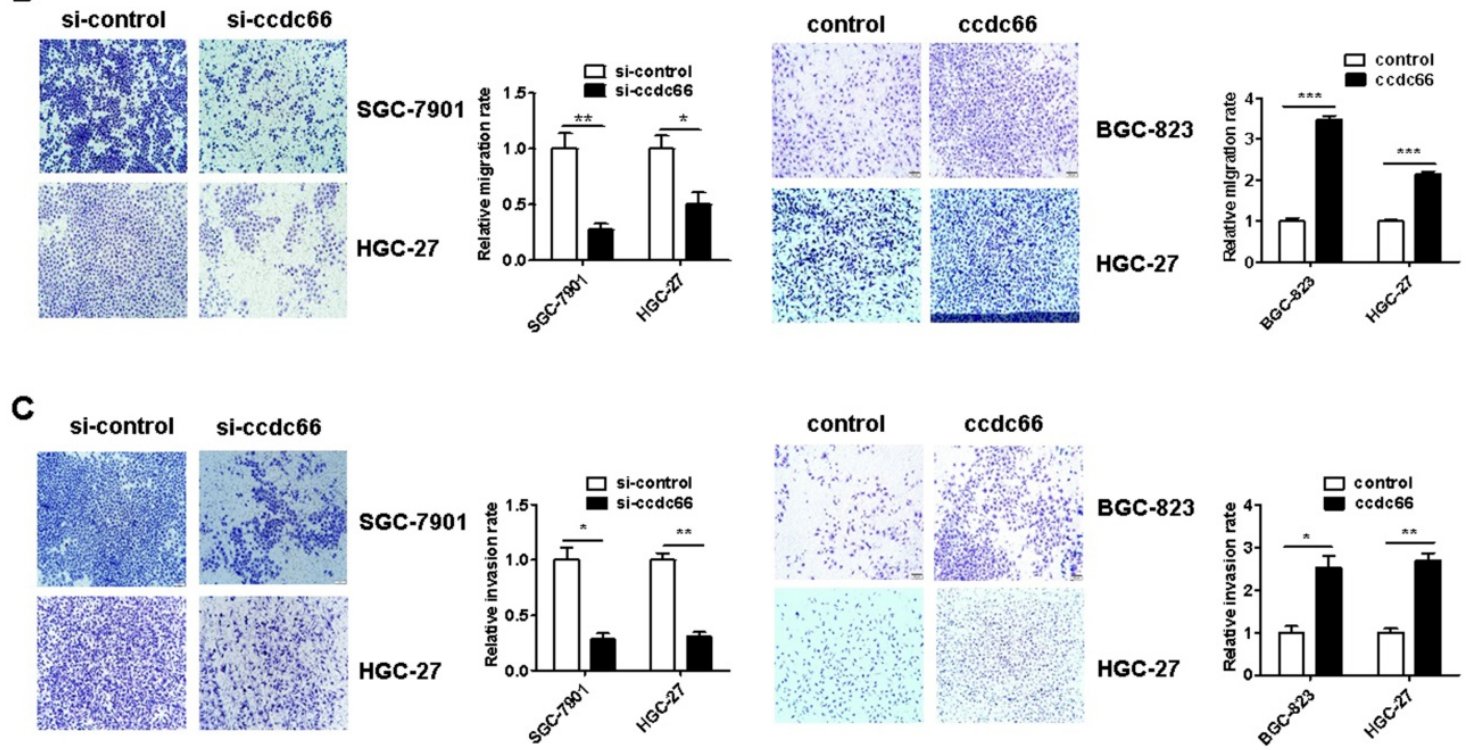

Figure 5 CircCCDC66 knockdown inhibits while overexpression promotes the migration and invasion of gastric cancer cells. (A) Wound healing assays for circCCDC66 siRNAs and overexpressing plasmid transfected GC cells. (B) Transwell migration assays for circCCDC66 siRNAs and overexpressing plasmid transfected GC cells. (C) Matrigel invasion assays for the invasive abilities of GC cells transfected with circCCDC66 siRNAs and overexpressing plasmid. All the experiments were performed in triplicate. $* P<0.05$, $* * P<0.01$, $* * * P<0.001$.

\section{c-Myc and TGF- $\beta 1$ knockdown abrogates the promotion of GC cell proliferation, migration, and invasion by circCCDC66.}

Since c-Myc is a powerful oncogene in cancer cell proliferation, we knocked down c-Myc in circCCDC66-overexpressing BGC-823 cells to see if it affects GC cell proliferation. We found that c-Myc knockdown remarkably reversed the promoting roles of circCCDC66 overexpression in GC cell proliferation (Figures 7A and 7B). The knockdown of c-Myc also inhibited the upregulation of cyclin D1, a downstream target of c-Myc, in circCCDC66-overexpressing GC cells (Figure 7C). To further demonstrate the importance of TGF- $\beta 1$ in the oncogenic roles of circCCDC66 in gastric cancer, we knocked down TGF- $\beta 1$ expression in circCCDC66-overexpressing BGC-823 and HGC-27 cells by using siRNA. Compared with circCCDC66-overexpressing group, TGF- $\beta 1$ knockdown impaired the promotion of GC cell migration and invasion by circCCDC66 (Figures $7 \mathrm{D}$ and $7 \mathrm{E})$. TGF- $\beta 1$ knockdown also inhibited the upregulation of p-SMAD2 and MMP9 in circCCDC66-overexpressing GC cells (Figure 7F). In summary, circCCDC66 could regulate GC cell proliferation, migration, and invasion by regulating c-Myc and TGF- $\beta$ signaling pathways. 


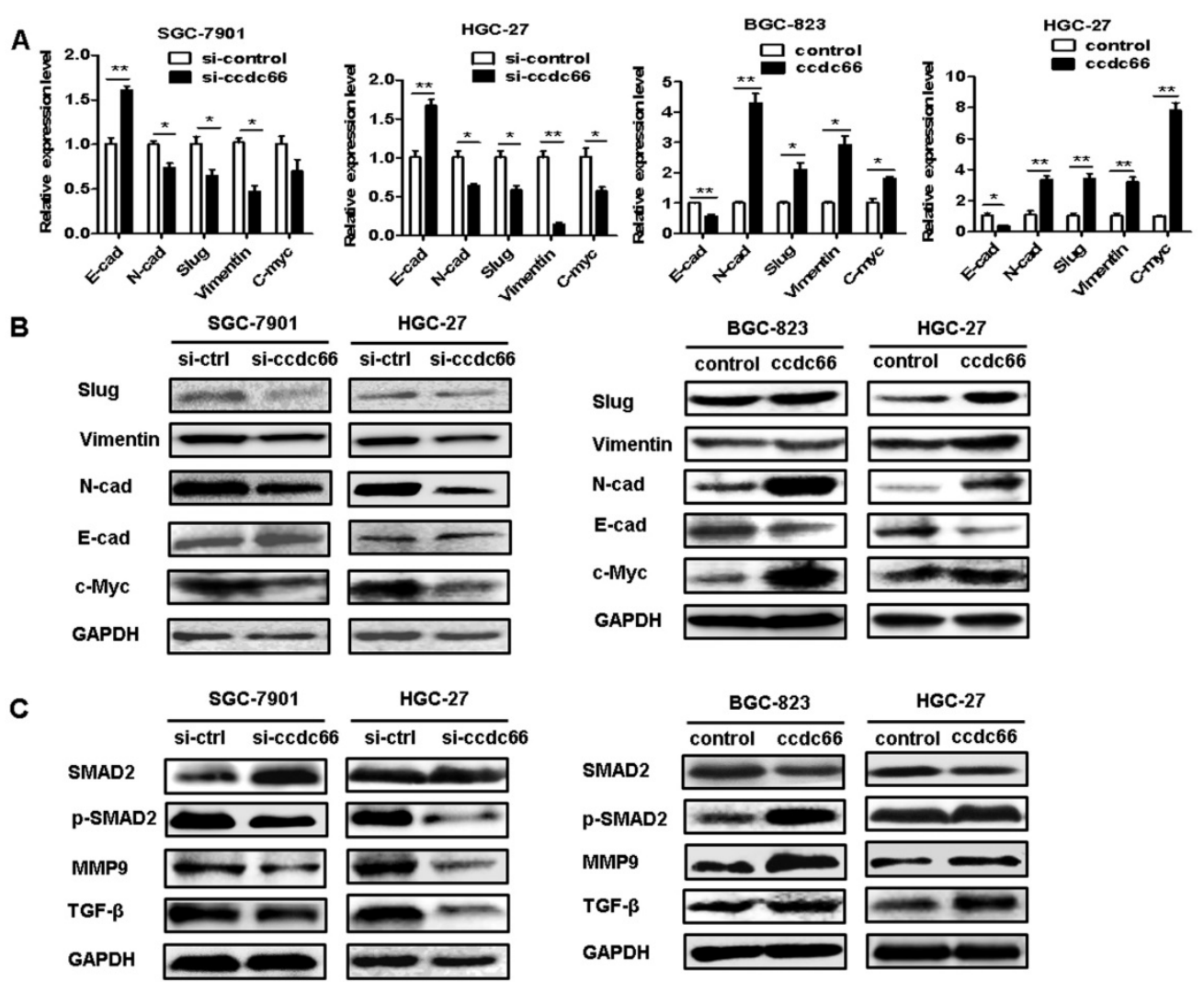

Figure 6 CircCCDC66 regulates c-Myc and TGF- $\beta$ signaling pathways in GC cells. (A) QRT- PCR and (B) Western blot analyses of EMT-specific markers in GC cells transfected with circCCDC66 siRNAs and overexpressing plasmid. (C) Western blot analysis of TGF- $\beta$, SMAD2, p-SMAD2 and MMP9 in GC cells transfected with circCCDC66 siRNAs and overexpressing plasmid. All the experiments were repeated for three times. $* P<0.05, * * P<0.01, * * * P<0.001$.

A

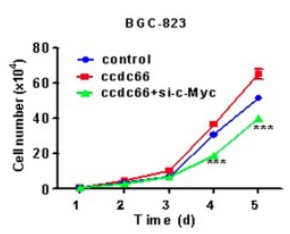

B

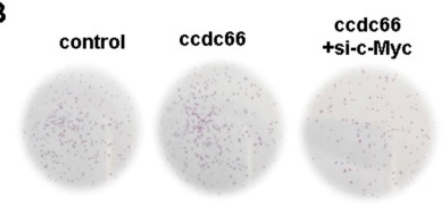

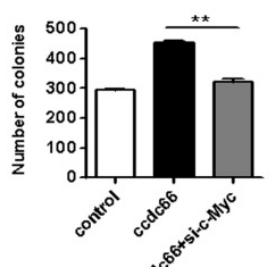

D

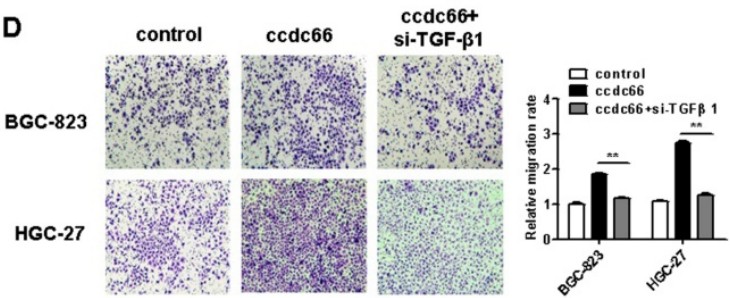

$\mathbf{F}$

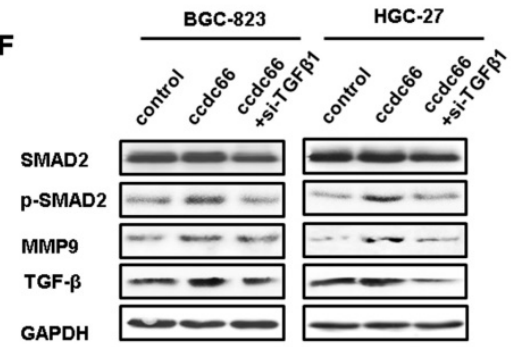

Figure 7 Inhibition of c-Myc and TGF- $\beta 1$ suppresses the promotion of GC cell proliferation, migration and invasion by circCCDC66. (A) Cell growth curves for circCCDC66-overexpressing GC cells with or without c-Myc knockdown. (B) The colony formation ability of circCCDC66-overexpressing GC cells with or without c-Myc knockdown. (C) Western blot analyses of c-Myc and cyclin DI expression in circCCDC66-overexpressing GC cells with or without c-Myc knockdown. (D) Transwell migration and (E) Matrigel invasion assays for the migration and invasion abilities of circCCDC66-overexpressing GC cells with or without TGF- $\beta 1$ knockdown. (F) Western blot analyses of TGF- $\beta$, p-SMAD2, and MMP9 expression in circCCDC66-overexpressing GC cells with or without TGF- $\beta 1$ knockdown. All the experiments were performed in triplicate. $* P<0.05$, $* * P<0.01$, $* * * P<0.001$, compared to circCCDC 66 group. 


\section{Discussion}

In recent years, circular RNAs have attracted great interest in cancer research field. Several circRNAs have been discovered to be aberrantly expressed in tumor tissues, plasma, serum and gastric juice of GC patients [30-32]. However, the expression pattern and biological function of circCCDC66 in GC have not been revealed. Herein, we reported that circCCDC66 was upregulated in GC tissues and cell lines, and its upregulation was significantly associated with tumor stage and lymphatic metastasis, suggesting a potential of circCCDC66 to be used as a marker for GC diagnosis. We further investigated the effects of circCCDC66 on gastric cancer progression and elucidated the underlying mechanisms. We found that depletion of circCCDC66 suppressed the proliferation, migration and invasion of GC cells but promoted the apoptosis of GC cells. CircCCDC66 overexpression showed the opposite effects. The results of in vivo study showed that the tumors in si-circCCDC66 group had smaller volumes and weights than that in si-control group, implicating that circCCDC66 is critically involved in GC progression.

CircRNAs have been identified as critical regulators of gene and protein expression in gastric cancer [33-37]. For instance, the expression of circOSBPL10 is upregulated in GC tissues and its level is a prognostic marker of the overall survival and disease-free survival of patients with GC. CircOSBPL10 promotes the growth and metastasis of gastric cancer by regulating miR-136-5p-WNT2 axis [38]. The expression of circAKT3 is higher in cisplatin (CDDP)-resistant GC tissues and cells than that in CDDP-sensitive samples. CircAKT3 promotes DNA damage repair and inhibits the apoptosis of GC cells by sponging miR-198 to upregulate PIK3R1 expression [39]. CircCCDC66 expression is first discovered to be elevated in colon cancer and is associated with poor prognosis [21]. CircCCDC66 controls cell proliferation, migration, and invasion via regulation of a subset of oncogenes. Recently, Wen et al. demonstrate that circCCDC66 targets DCX to regulate cell proliferation and migration by sponging miR-488-3p in Hirschsprung's disease [40]. Recently, Yang et al. suggest that circCCDC66 knockdown attenuates the proliferative and invasive abilities of GC cells by regulating miR-1238-3p/LHX2 axis [41]. In consistent with these findings, we reported that circCCDC66 was upregulated in GC tissues and cells and circCCDC66 overexpression promoted while circCCDC66 knockdown inhibited GC cell proliferation, migration and invasion, suggesting that circCCDC66 plays an oncogenic role in GC.
EMT is closely associated with tumor progression and metastasis and is regulated by various signaling pathways [42]. The previous studies have shown that circRNAs could induce EMT to promote tumor progression. For example, $\mathrm{Li}$ and colleagues demonstrate that cir-ITCH inactivates wnt/ $\beta$-catenin pathway to inhibit esophageal squamous cell carcinoma [43]. Recently, Zhou et al. suggest that circRNA_0023642 could promote GC cell migration and invasion through the regulation of EMT [44]. In this study, we showed that circCCDC66 knockdown inhibited the expression of TGF- $\beta$ and inactivated SMAD signaling pathway, thus reversing EMT in GC cells, suggesting that circCCDC66 is an important regulator of EMT in gastric cancer. We also found that circCCDC66 knockdown decreased while circCCDC66 overexpression increased the expression of c-Myc in GC cells. Our results are consistent with that reported by Hsiao et al. showing that circCCDC66 could upregulate c-Myc expression by acting as miRNA sponge for miR-33b and miR-93 [21]. However, the mechanism by which circCCDC66 regulate c-Myc and TGF- $\beta$ signaling pathways in GC cells is currently unknown and deserves further investigation.

\section{Conclusions}

In summary, circCCDC66 was upregulated in GC tissue and its expression level was significantly associated with tumor progression. CircCCDC66 knockdown inhibited while circCCDC66 overexpression promoted GC cell proliferation, migration and invasion. CircCCDC66 knockdown induced GC cell apoptosis and suppressed tumor growth. CircCCDC66 played oncogenic roles in GC via the activation of $\mathrm{c}-\mathrm{Myc}$ and TGF- $\beta$ signaling pathways.

\section{Abbreviations}

CircRNAs: circular RNAs; circCCDC66: circular RNA CCDC66; CRC: colorectal cancer; EMT: epithelial to mesenchymal transition; GC: gastric cancer; HCC: hepatocellular cancer; RBPs: RNA binding proteins.

\section{Supplementary Material}

Supplementary figures and tables. http://www.jcancer.org/v11p2759s1.pdf

\section{Acknowledgments}

This work was supported by the National Natural Science Foundation of China (81972310, 81672416, 81572075), Major Natural Science Reasearch Project for Universities in Jiangsu Province (18KJA320001), Key Laboratory of Molecular 
Diagnostics and Precision Medicine for Surgical Oncology in Gansu Province (2019GSZDSYS01), and Priority Academic Program Development of Jiangsu Higher Education Institutions (PAPD).

\section{Competing Interests}

The authors have declared that no competing interest exists.

\section{References}

1. Bray F, Ferlay J, Soerjomataram I, Siegel RL, Torre LA, Jemal A. Global cancer statistics 2018: GLOBOCAN estimates of incidence and mortality worldwide for 36 cancers in 185 countries. CA Cancer J Clin. 2018; 68: 394-424.

2. Huang YS, Jie N, Zou KJ, Weng Y. Expression profile of circular RNAs in human gastric cancer tissues. Mol Med Rep. 2017;16: 2469-2476.

3. Karimi P, Islami F, Anandasabapathy S, Freedman ND, Kamangar F. Gastric cancer: descriptive epidemiology, risk factors, screening, and prevention. Cancer Epidemiol Biomarkers Prev. 2014; 23(5): 700-13.

4. Hsiao KY, Sun HS, Tsai SJ. Circular RNA - New member of noncoding RNA with novel functions. Exp Biol Med (Maywood). 2017; 242: 1136-1141.

5. Mitra A, Pfeifer K, Park KS. Circular RNAs and competing endogenous RNA (ceRNA) networks. Transl Cancer Res. 2018; 7: S624-S628.

6. Li Z, Huang C, Bao C, Chen L, Lin M, Wang X, et al. Exon-intron circular RNAs regulate transcription in the nucleus. Nat Struct Mol Biol. 2015; 22(3):256-264

7. Zhang F, Zhang R, Zhang X, Wu Y, Li X, Zhang S, et al. Comprehensive analysis of circRNA expression pattern and circRNA-miRNA-mRNA network in the pathogenesis of atherosclerosis in rabbits. Aging (Albany NY). 2018; 10: 2266-2283.

8. Yang $\mathrm{Y}$, Fan X, Mao M, Song X, Wu P, Zhang Y, et al. Extensive translation of circular RNAs driven by N6-methyladenosine. Cell Res. 2017; 27:626-641.

9. Bazan HA, Hatfield SA, Brug A, Brooks AJ, Lightell DJ Jr, Woods TC. Carotid plaque rupture is accompanied by an increase in the ratio of serum circR-284 to miR-221 levels. Circ Cardiovasc Genet. 2017; 10:e001720.

10. Li J, Lin H, Sun Z, Kong G, Yan X, Wang Y, et al. High-throughput data of circular RNA profiles in human temporal cortex tissue reveals novel insights into temporal lobe epilepsy. Cell Physiol Biochem. 2018; 45: 677-691.

11. Zhao Y, Alexandrov PN, Jaber V, Lukiw WJ. Deficiency in the ubiquitin conjugating enzyme UBE2A in Alzheimer's disease (AD) is linked to deficits in a natural circular miRNA-7 sponge (circRNA; ciRS-7). Genes. 2016; 7:116

12. Fang Y, Wang X, Li W, Han J, Jin J, Su F, et al. Screening of circular RNAs and validation of circANKRD36 associated with inflammation in patients with type 2 diabetes mellitus. Int J Mol Med. 2018; 42: 1865-1874.

13. Vidal AF, Ribeiro-Dos-Santos AM, Vinasco-Sandoval T, Magalhães L, Pinto $P$, Anaissi AKM, et al. The comprehensive expression analysis of circular RNAs in gastric cancer and its association with field cancerization. Sci Rep. 2017; 7: 14551.

14. Chen G, Wang $Q$, Yang $Q$, Li Z, Du Z, Ren M, et al. Circular RNAs hsa_circ_0032462, hsa_circ_0028173, hsa_circ_0005909 are predicted to promote CADM1 expression by functioning as miRNAs sponge in human osteosarcoma. PLoS One. 2018; 13: e0202896.

15. Yu W, Jiang H, Zhang H, Li J. Hsa_circ_0003998 promotes cell proliferation and invasion by targeting miR-326 in non-small cell lung cancer. Onco Targets Ther. 2018; 11: 5569-5577.

16. Ashwal-Fluss R, Meyer M, Pamudurti NR, Ivanov A, Bartok O, Hanan M, et al. circRNA biogenesis competes with pre-mRNA splicing. Mol Cell. 2014; 56:55-66.

17. Abdelmohsen K, Panda AC, Munk R, Grammatikakis I, Dudekula DB, De S, et al. Identification of HuR target circular RNAs uncovers suppression of PABPN1 translation by CircPABPN1. RNA Biol. 2017; 14(3):361-369.

18. Hansen TB, Jensen TI, Clausen BH, Bramsen JB, Finsen B, Damgaard CK, et al. Natural RNA circles function as efficient microRNA sponges. Nature. 2013; 495(7441): 384-8

19. $\mathrm{Yu} \mathrm{H}$, Chen $\mathrm{Y}$, Jiang $\mathrm{P}$. Circular RNA HIPK3 exerts oncogenic properties through suppression of miR-124 in lung cancer. Biochem Biophys Res Commun. 2018; 506(3): 455-462.

20. Han D, Li J, Wang H, Su X, Hou J, Gu Y, et al. Circular RNA circMTO1 acts as the sponge of microRNA-9 to suppress hepatocellular carcinoma progression. Hepatology. 2017; 66: 1151-1164.

21. Hsiao KY, Lin YC, Gupta SK, Chang N, Yen L3 Sun HS, et al. Noncoding effects of circular RNA CCDC66 promote colon cancer growth and metastasis. Cancer Res. 2017; 77: 2339-2350.

22. Huang HR, Wei L, Qin T, Yang N, Li Z, Xu Z. Circular RNA ciRS-7 triggers the migration and invasion of esophageal squamous cell carcinoma via miR-7/KLF4 and NF-kappaB signals. Cancer Biol Ther. 2019; 20:73-80.

23. Zhang HD, Jiang LH, Hou JC, Zhong SL, Zhou SY, Zhu LP, et al. Circular RNA hsa_circ_0052112 promotes cell migration and invasion by acting as sponge for miR-125a-5p in breast cancer. Biomed Pharmacother. 2018; 107: 1342-1353.
24. He JH, Li YG, Han ZP, Zhou JB, Chen WM, Lv YB, et al. The CircRNA-ACAP2/Hsa-miR-21-5p/Tiam1 regulatory feedback circuit affects the proliferation, migration, and invasion of colon cancer SW480 cells. Cell Physiol Biochem. 2018; 49: 1539-1550.

25. Zhang Y, Li J, Yu J, Liu H, Shen Z, Ye G, et al. Circular RNAs signature predicts the early recurrence of stage III gastric cancer after radical surgery. Oncotarget. 2017; 8: 22936-22943.

26. Sun HD, Tang WW, Rong DW, Jin H, Fu K, Zhang W, et al. Hsa_circ_0000520, a potential new circular RNA biomarker, is involved in gastric carcinoma. Cancer Biomark. 2018; 21: 299-306.

27. Zhang J, Liu H, Hou L, Wang G, Zhang R, Huang Y, et al. Circular RNA_LARP4 inhibits cell proliferation and invasion of gastric cancer by sponging miR-424-5p and regulating LATS1 expression. Mol Cancer. 2017; 16: 151.

28. Liu H, Liu Y, Bian Z, Zhang J, Zhang R, Chen X, et al. Circular RNA YAP1 inhibits the proliferation and invasion of gastric cancer cells by regulating the miR-367-5p/p27 (Kip1) axis. Mol Cancer. 2018; 17: 151.

29. Yang F, Fang E, Mei H, Chen Y, Li H, Li D, et al. Cis-Acting circ-CTNNB1 promotes beta-Catenin signaling and cancer progression via DDX3-Mediated transactivation of YY1. Cancer Res. 2019; 79: 557-571.

30. Zhao Q, Chen S, Li T, Xiao B, Zhang X. Clinical values of circular RNA 0000181 in the screening of gastric cancer. J Clin Lab Anal. 2018; 32: e22333.

31. Li WH, Song YC, Zhang H, Zhou ZJ, Xie X, Zeng QN, et al. Decreased expression of hsa_circ_ 00001649 in gastric cancer and its clinical significance. Dis Markers. 2017; 2017: 1-6.

32. Shao Y, Li J, Lu R, Li T, Yang Y, Xiao B, et al. Global circular RNA expression profile of human gastric cancer and its clinical significance. Cancer Med. 2017; 6: $1173-1180$

33. Ding L, Zhao Y, Dang S, Wang Y, Li X, Yu X, et al. Circular RNA circ-DONSON facilitates gastric cancer growth and invasion via NURF complex dependent activation of transcription factor SOX4. Mol Cancer. 2019; 18:45.

34. Rong D, Lu C, Zhang B, Fu K, Zhao S, Tang W, et al. CircPSMC3 suppresses the proliferation and metastasis of gastric cancer by acting as a competitive endogenous RNA through sponging miR-296-5p. Mol Cancer. 2019; 18:25.

35. Zhang $X$, Wang $S$, Wang $H$, et al. Circular RNA circNRIP1 acts as a microRNA-149-5p sponge to promote gastric cancer progression via the AKT1/mTOR pathway. Mol Cancer. 2019; 18:20

36. Fang J, Hong $\mathrm{H}$, Xue $\mathrm{X}$, Zhu $\mathrm{X}$, Jiang $\mathrm{L}$, Qin $\mathrm{M}$, et al. A novel circular RNA, circFAT1(e2), inhibits gastric cancer progression by targeting miR-548g in the cytoplasm and interacting with YBX1 in the nucleus. Cancer Lett. 2019; 442.222-232.

37. Chen Y, Yang F, Fang E, Xiao W, Mei H, Li H, et al. Circular RNA circAGO2 drives cancer progression through facilitating HuR-repressed functions of AGO2-miRNA complexes. Cell Death Differ. 2019; 26:1346-1364.

38. Wang S, Zhang X, Li Z, Wang W, Li B, Huang X, et al. Circular RNA profile identifies circOSBPL10 as an oncogenic factor and prognostic marker in gastric cancer. Oncogene. 2019; 38:6985-7001.

39. Huang X, Li Z, Zhang Q, Wang W, Li B, Wang L, et al. Circular RNA AKT3 upregulates PIK3R1 to enhance cisplatin resistance in gastric cancer via miR-198 suppression. Mol Cancer. 2019; 18:71.

40. Wen Z, Shen Q, Zhang H, Su Y, Zhu Z, Chen G, et al. Circular RNA CCDC66 targets DCX to regulate cell proliferation and migration by sponging miR-488-3p in Hirschsprung's disease. J Cell Physiol. 2019; 234:10576-10587.

41. Yang M, Wang GY, Qian H, Ji XY, Liu CY, Zeng XH, et al. Circ-CCDC66 accelerates proliferation and invasion of gastric cancer via binding to miRNA-1238-3p. Eur Rev Med Pharmacol Sci. 2019; 23:4164-4172.

42. Xu Q, Deng F, Qin Y, Zhao Z, Wu Z, Xing Z, et al. Long non-coding RNA regulation of epithelial-mesenchymal transition in cancer metastasis. Cell Death Dis. 2016; 7: e2254

43. Li F, Zhang L, Li W, Deng J, Zheng J, An M, et al. Circular RNA ITCH has inhibitory effect on ESCC by suppressing the Wnt/beta-catenin pathway. Oncotarget. 2015; 6: 6001-13.

44. Zhou LH, Yang YC, Zhang RY, Wang P, Pang MH, Liang LQ. CircRNA 0023642 promotes migration and invasion of gastric cancer cells by regulating EMT. Eur Rev Med Pharmacol Sci. 2018; 22: 2297-2303. 